\title{
EDWYN REED Y SU CONTRIBUCIÓN A LA FORMACIÓN DE COLECCIONES DE HISTORIA NATURAL EN CHILE ${ }^{1}$
}

\section{EDWYN REED AND HIS CONTRIBUTION TO THE FORMATION OF NATURAL HISTORY COLLECTIONS IN CHILE}

\author{
Carolina Valenzuela Matus \\ Universidad Autónoma de Chile \\ Galvarino Gallardo $1973,2^{\circ}$ piso, Providencia, Santiago \\ carolina.valenzuela01@uautonoma.cl
}

\begin{abstract}
Resumen
El naturalista inglés Edwyn Reed (1841-1910) contribuyó significativamente a la formación de colecciones de historia natural en Chile, en el contexto de la organización de los primeros museos de este tipo en el país. Este artículo se propone analizar tres aspectos que evidencian este aporte; primero, su labor como naturalista en Chile, desarrollada en la senda marcada por otros científicos extranjeros como Claudio Gay o Rodulfo
\end{abstract}

Este artículo es fruto del proyecto Fondecyt 11170033, “Antigüedades y naturaleza: circulación interoceánica de objetos en los primeros gabinetes de historia natural como estrategia de posicionamiento de la ciencia en Chile”. Agradezco al Museo de Historia Natural de Concepción, muy especialmente a Marco Sánchez Aguilera y a Roxana Torres Rossel. Sumo a mis agradecimientos a Gina Inostroza Retamal, por su valiosa colaboración en la búsqueda de documentos. 
Philippi; segundo, su trabajo en la enseñanza de las ciencias y en la confección de gabinetes de historia natural para los liceos del país y, por último, su papel como director del Museo de Historia Natural de Concepción y gestor de las primeras colecciones de la institución. En estos tres ámbitos se percibe el aporte de Reed al desarrollo de las ciencias naturales en Chile y a la consolidación de redes globales de colaboración que fortalecieron los museos de Santiago, Valparaíso y Concepción.

Palabras clave: Museo Nacional, Museo de Concepción, Museo de Valparaíso, naturalistas, colecciones naturales, gabinetes, Edwyn Reed.

\begin{abstract}
The English naturalist Edwyn Reed (1841-1910) made an important contribution to the formation of Natural History collections in Chile, in the context of the creation of the first museums of Natural History in the country. This article analyzes three aspects of his legacy: first, his work as a naturalist, developed from the work previously done by other foreign scientists such as Claude Gay or Rudolph Philippi; second, his work in making cabinets of Natural History for Public High Schools (Liceos) in Chile; and third, his role as the director of Natural History Museum of Concepción and manager of the first collections of the institution. These three aspects reveal Edwyn Reed's contribution to the development of the natural sciences in Chile and to the consolidation of a collaborative global network that strengthened the museums of Santiago, Valparaíso, and Concepción.
\end{abstract}

Keywords: National Museum, Concepción Museum, Valparaíso Museum, Naturalists, Natural Collections, Cabinets, Edwyn Reed.

Recibido: 09/12/2019

Aceptado: 09/01/2020 
Las ciencias cobraron una progresiva importancia en Chile a lo largo del siglo XIX, un país de reciente formación que requería de expertos naturalistas para llevar a cabo un reconocimiento exhaustivo de la naturaleza, de la geografía y de los recursos naturales del territorio. Esta actividad vino acompañada con la formación de los museos de historia natural en el país, instituciones que paulatinamente se convirtieron en importantes centros de conocimiento, intercambio y desarrollo científico. En este contexto, el naturalista inglés Edwyn Reed desarrolló un extenso y destacado trabajo en el avance de las ciencias naturales. Parte de su labor se vinculó a la formación de colecciones en los museos de historia natural y gabinetes para la enseńanza de las ciencias naturales en el país a fines del siglo XIX y principios del XX.

Aunque la figura de Edwyn Reed no es del todo desconocida, su biografía y el estudio sobre su trabajo científico solo se conocen parcialmente. Lo que en gran parte sabemos de su labor como naturalista se debe a sus publicaciones en los Anales de la Universidad de Chile y en la Revista Chilena de Historia Natural, mientras que sus aspectos biográficos los conocemos gracias a escritos como el de Carlos Porter "Galería de naturalistas chilenos”, publicado en la Revista Chilena de Historia Natural en 1903; el libro Apuntes para la historia del Museo de Concepción (1911) escrito por su hijo, Carlos Reed o posteriores contribuciones como la biografía novelada de Walter Reed: Edwyn C. Reed y el archivador rojo (2013). Sin embargo, se requieren trabajos más amplios que pongan en valor la contribución de este naturalista a la ciencia del país. En este texto, no pretendo mostrar al científico inglés como un sujeto aislado ni menos perpetuar la visión de una ciencia heroica, que narra la historia de los grandes personajes. Este artículo destaca el trabajo de Reed en el campo científico de su época, valorando su papel dentro del fenómeno de la ciencia transnacional que surge con la migración de científicos europeos quienes contribuyen a la formación de establecimientos dedicados a la ciencia (Vilo y Sanhueza).

Para aproximarme a los aspectos más relevantes de la contribución científica de Reed, analizo, en primer lugar y como contexto general, los inicios de los museos de historia natural en Chile, aspecto esencial, ya que 
Reed trabajó en todos ellos, así como las influencias intelectuales recibidas por el naturalista inglés, para de este modo examinar su aporte a la formación de gabinetes de historia natural y a la creación del Museo de Concepción en 1902. Hay que considerar, sobre todo, que la labor de Edwyn Reed en Chile fue prolífica y se nutrió de su participación en redes colaborativas globales establecidas con otros naturalistas e instituciones, tanto en Chile como en el extranjero. En esta red, los naturalistas se conectaban con sus pares de otras latitudes para el intercambio de conocimientos y objetos, así como también para la recolección, la venta, el canje y la donación de piezas y libros. Esto permitió la formación de colecciones en los museos de historia natural del país, y favoreció la elaboración de gabinetes para la enseñanza de las ciencias naturales en los liceos de Chile.

En este estudio, se identifican al menos tres aspectos que reflejan su contribución: el primero, su labor como naturalista, desarrollada en la senda marcada por otros científicos extranjeros en Chile; el segundo, el impulso que dio a la enseñanza de las ciencias naturales y a la creación de gabinetes de historia natural de confección nacional para los liceos, y, el tercero, su gestión como director del Museo de Historia Natural de Concepción y gestor de las primeras colecciones de la institución, en un momento clave para la formación de museos estatales y la consolidación de las ciencias naturales en Chile.

\section{LOS INICIOS DE LOS MUSEOS DE HISTORIA NATURAL EN Chile}

A principios del siglo XIX, la historia natural era un campo de incipiente interés en Chile. El mayor referente de esta disciplina en la época era el abate Juan Ignacio Molina, quien escribió desde el exilio jesuita su Saggio sulla Storia Naturale del Chili, editado en Bolonia en 1782. En cuanto a la formación de gabinetes de historia natural, pareciera que el interés por el acopio de objetos habría ocurrido de forma más tardía en Chile que en otros territorios de la América española, como Perú y México. De acuerdo 
con Podgorny y Lopes, los primeros museos de historia natural de la América española se atribuyen a las fundaciones de José Longinos Martínez (1750-1803), miembro de la Real Expedición Botánica a la Nueva España, así como al obispo de la Intendencia de Trujillo (Perú), Jaime Martínez Compañón (1737-1797), quien formó otro importante gabinete de flora, fauna, metales, minerales y antigüedades (Podgorny y Lopes 35). En Chile, a fines del período colonial, se evidencia cierto impulso en la recolección de objetos gracias a la creación del gabinete de minerales de la Real Academia de San Luis en 1803. Este habría sido estimulado por Manuel de Salas convencido de la necesidad urgente de promover la minería "a través del fomento de su enseńanza, la cual se hallaba en franco deterioro a pesar de la importancia para la economía de Chile colonial" (Laboulais citado en Serra, "Minerales" 160). El gabinete de minerales continuó alojado en la Academia hasta su incorporación al Instituto Nacional en 1813 (160). Tras la consolidación del proceso de independencia, Bernardo O'Higgins, director supremo de la República en 1818, también concibió la idea de reunir en un museo muestras de las producciones naturales de Chile y encargó esta misión a Juan José Dauxion Lavaysse, sin ningún éxito (Philippi 4).

En la década de 1830, el gobierno chileno contrató a importantes científicos extranjeros para el estudio del territorio. En 1830, se comisionaba al naturalista francés Claudio Gay (1800-1873) para ciertas exploraciones, en el contexto de la organización del nuevo Estado nación, lo que le permitió escribir su monumental obra Historia física y politica de Chile. Ocho años después, se contrataba al profesor polaco Ignacio Domeyko (1802-1889) para enseñar química y mineralogía en el Liceo de Coquimbo con el propósito de fomentar el desarrollo minero. Domeyko hizo varias expediciones por el territorio nacional y promovió el conocimiento geológico del país. Años más tarde, en 1848, el gobierno chileno requirió los servicios del geólogo francés José Amado Pissis, para llevar a cabo un estudio geológico, topográfico y mineralógico, y hacer un reconocimiento del desierto de Atacama y la descripción geológica y mineralógica de la república. Todas estas exploraciones del territorio tenían por objetivo sistematizar información relevante de los recursos naturales del país. 
Los viajes de Claudio Gay y los materiales que recopiló tuvieron un importante impacto en la formación de un primer gabinete de historia natural en Santiago. Hasta 1838, Gay estuvo casi exclusivamente centrado en la recolección de objetos naturales, participando también en activas redes de intercambio interoceánico como corresponsal del Museo de Historia Natural de París gracias a su práctica en la búsqueda, acopio y preparación de especímenes. De acuerdo con Daniela Serra, mientras el científico recorría diferentes rincones del país investigando y reuniendo ejemplares de historia natural, una comisión científica era nombrada por las autoridades para examinar sus trabajos ("La configuración" 56). Esta comisión estaba compuesta por Vicente Bustillos, Alejo Bezanilla y Francisco García Huidobro, quienes se encargaron de almacenar y cuidar las colecciones que arribaban a la capital, al mismo tiempo que se gestionó un espacio e infraestructura para la preparación y exhibición de los ejemplares naturales. En este sentido, afirma Serra que, contrariamente a lo que se ha señalado hasta ahora, la instalación y organización del gabinete de historia natural que debía fundarse en el centro de la ciudad no fue una obra exclusiva del francés, sino el resultado de un trabajo colectivo característico del quehacer científico natural en el Chile de 1830 (72).

Pese a ello, tras el regreso de Gay a Francia en 1842, se fue deteriorando el gabinete. El impulso para su mantenimiento fue retomado por el naturalista alemán Rodulfo Amando Philippi (1808-1904) quien lo elevó a la categoría de Museo Nacional, asumiendo el cargo de director en 1853. De acuerdo con Carlos Sanhueza, "la llegada de Philippi supuso un reordenamiento y una profesionalización del Museo, crea una cultura monográfica, transforma un conjunto de objetos en una entidad al servicio de la investigación científica en Chile" (“Coleccionismo" 173). Este trabajo de consolidación de la institución seguiría afianzándose en los años venideros y continuaría con su hijo, Federico Philippi (1838-1910), quien lo reemplazó como director. 


\section{La labor de Edwyn Reed en Chile}

Desde 1869 a 1877, Rodulfo Philippi contó en el Museo Nacional con la colaboración del entomólogo Edwyn Ch. Reed Brockmann (1841-1910), primero como asistente y luego como ayudante. Este joven inglés, procedente de Bristol, ya tenía una experiencia destacable, pues en su ciudad natal ocupó el cargo de secretario ad honorem de la Sociedad Entomológica y de la Sociedad Microscópica, al mismo tiempo que fue ayudante del Museo de Ciencias Naturales (W. Reed 23). También estaba familiarizado con la naturaleza de Sudamérica, ya que, comisionado por el gobierno británico e inspirado por la labor naturalista de Charles Darwin (Porter 138), había pasado tres años en Brasil estudiando la fauna entomológica, lo que le sirvió para complementar la clasificación hecha por Gay, identificando nuevas especies. En la misma época, también contribuyeron los jóvenes estudiantes Vicente Izquierdo y Benjamín Dávila al borrador de los catálogos de los insectos indígenas existentes en el Museo Nacional (Carta de Philippi al Ministro de Instrucción Pública, 13 de junio de 1869), lo que nos indica que estas tareas de clasificación no se realizaron aisladamente, sino que requieren de un trabajo colaborativo donde se integre el conocimiento de diversos participantes y disciplinas.

Además de las labores de clasificación entomológica, Reed se dedicó a preparar especímenes para canjes con museos del extranjero, tal como informa Rodulfo Philippi al Ministro de Instrucción Pública.

El señor Reed ha seguido clasificando los insectos extranjeros, que había recibido en varias ocasiones, y habían quedado, hasta ahora en los mismos cajones que habían venido. Ahora está preparando un envío para California en canje de los insectos de esa interesante provincia que nos han sido prometidos y que podemos esperar de un día a otro. (Carta de Philippi al Ministerio de Instrucción Pública, 3 de agosto de 1870)

Los viajes de exploración y recolección de especies eran parte importante del trabajo de los naturalistas en el país. En 1872, Rodulfo 
Philippi envió a Edwyn Reed a una misión científica a isla Mocha (actual región del Biobío), ya que le llegaron noticias de que ahí se había descubierto el sándalo, hecho curioso al tratarse de una especie de palo proveniente de la zona tórrida. De acuerdo con las cartas de Philippi, el viaje de Reed fue bastante accidentado, pues para llegar a la isla, el naturalista tuvo que seguir viaje hacia Corral (desembocadura del río Valdivia, actual región de los Ríos), único lugar donde pudo conseguir que un vapor lo llevara. Mientras esperaba en Valdivia, Reed aprovechó el tiempo para recoger "un buen número de cuero de aves, lobos marinos y de plantas" (Carta de Philippi al Ministerio de Instrucción Pública, 13 de mayo de 1872).

Una vez en isla Mocha, Reed se dedicó a conocer más sobre la naturaleza del lugar y sus producciones. Descubrió que el sándalo del que les llegaron noticias no era tal sino un arbustito con flores, llamado Siete camisas (escallonia macrantha). De acuerdo con Philippi, Reed halló entre los insectos de la isla "varios muy interesantes y aún nuevos para la ciencia" (Carta de Philippi al Ministro de Instrucción Pública, 13 de mayo de 1872). El descubrimiento y clasificación de especies comenzada años antes por Gay tuvo continuidad en este viaje exploratorio.

Por otra parte, Reed actuó, al igual que el naturalista francés, como agente en la adquisición de colecciones para el Museo Nacional en París y Londres. En 1873, se le encargó cotejar un número de insectos y otros animales chilenos con los tipos que existían en las grandes colecciones de esas ciudades. Asimismo, estudió la organización de los principales museos de Europa, experiencia que le sirvió en la posterior organización de museos de historia natural en distintas regiones, además de fortalecer los contactos y redes internacionales que facilitaron el intercambio de especies. Pese a este camino de éxitos, Reed vivió una delicada situación que terminó por alejarlo del Museo Nacional:

Philippi se vio obligado a solicitar su renuncia debido a un caso de negocios ilícitos de "ventas de objetos de historia natural" a Inglaterra, evitándole así la deshonra que provocaría un decreto de destitución por parte del ministerio [...] Esta situación es similar a la que pasó anteriormente con el taxidermista 
Filiberto Germain, quien fue obligado a renunciar en 1858 por mantener negocios paralelos de venta de colecciones de ciencias naturales, además de entrar en conflicto de autoridad con Philippi por discusiones curatoriales. (Schell citada en Garrido 4)

En los documentos oficiales enviados al Ministerio de Instrucción Pública no se han encontrado referencias a este episodio. No obstante, algunos malentendidos también podrían haber influido en el alejamiento entre Philippi y Reed. El director del Museo Nacional hizo saber al Ministerio de Instrucción Pública la molestia que le causó que, en un decreto oficial, apareciera el nombre de Reed asociado al cargo de subdirector del museo, cuando era ocupado por Ludwig Landbeck. Asimismo, demuestra que Reed "pasa en los ojos de los Ingleses y aún de otras personas no solo como subdirector sino aún como director del museo" (Carta de Philippi al Ministro de Instrucción Pública, 24 de octubre de 1876). Lo siguiente que sabemos es que el 18 de mayo de 1877, Philippi comunica que "el Museo ha perdido la cooperación del Sr. Reed a quien estuvo confiado el cuidado de los insectos [...] pero tengo ahora la asistencia de los señores Luis Sanfurgo, Federico Puga y Enrique Ivar, nombrados auxiliares por decreto Supremo con cuyos trabajos estoy satisfecho" (Carta de Philippi al Ministro de Instrucción Pública, 18 de mayo de 1877).

En este conflicto personal podrían haber influido varios aspectos. Uno de ellos es que los naturalistas debían posicionar su nombre y fortalecer constantemente sus redes nacionales e internacionales para comprar, vender e intercambiar especímenes, recordando que "en la medida en que la comunidad científica reconocía un nombre, el intercambio de objetos podía hacerse sobre bases legitimadas y validadas" (Sanhueza, "Objetos" 159). Por tanto, la circulación de objetos desde el museo a otros extranjeros resultó una práctica frecuente. Por otra parte, no es de extrañar que pudiera generarse una importante competencia interna en la formación de redes prestigiosas y en la circulación de objetos, sin desconocer las posibles irregularidades de estos intercambios, no tenemos mayor información en las fuentes oficiales. Estos episodios deben analizarse, siguiendo a Alberti, 
como parte del relato de un museo que incluye la historia de la gente y de sus relaciones, de todos aquellos esfuerzos que sostienen la institución y sus colecciones, pero también de sus conflictos (150).

A pesar de este traspié, Reed tuvo la oportunidad de seguir en la formación de museos y también en la enseñanza. En 1878, era profesor de ciencias naturales en el Liceo de Valparaíso, período en que Reed inicia un trabajo prolífico a nivel regional. Sin embargo, ya con anterioridad, en 1875, había solicitado permiso para formar, sin remuneración, el Museo de Historia Natural en los Baños de Cauquenes (W. Reed 26) ${ }^{2}$. En 1878, junto con Eduardo de la Barra, rector del Liceo de Hombres de Valparaíso, Reed comenzó el arreglo del museo que se abriría en el puerto, del cual fue su primer director. Ese mismo año, formó el Museo de Historia Natural del Seminario San Rafael (Porter 13) y montó también un observatorio astronómico. Asimismo, impartió clases de historia natural y geografía física en la Escuela Naval (W. Reed 30). Los años en que estuvo involucrado en la formación de museos de historia natural le dieron experiencia y consolidaron sus redes de intercambio de conocimientos y objetos con museos e instituciones alrededor del globo. Estas redes fueron esenciales para la formación de colecciones, nutridas por la compra de objetos, pero también por el canje y las donaciones. En 1887, Reed se vio forzado a dejar Valparaíso; una malaria contraída en Brasil afectó su salud y lo obligó a buscar climas secos, como Los Andes y Rancagua, lugares que fueron su residencia antes de su traslado a Concepción.

\section{Creación de gabinetes para los liceos de Chile}

El naturalista inglés se adhería a un pensamiento muy extendido en el siglo XIX: las prácticas coleccionistas y el estudio de la historia natural eran beneficiosos para forjar el carácter del individuo, cultivando la

$2 \quad$ Walter Reed señala que "hoy no se trata de un museo propiamente tal, exclusivamente se conservan algunas muestras de la fauna local en el departamento de Ciencias Naturales en el Liceo de Cauquenes" (59). 
simplicidad y el autocontrol (Schell 20). De esta manera, la práctica de la historia natural se vinculaba a la formación de valores en los estudiantes. En Europa, las ciencias naturales también se habían convertido en una disciplina importante en la enseńanza oficial. Desde el siglo XIX, los institutos madrileños adquirían material pedagógico en París, ya que la capital francesa "contaba con numerosos establecimientos privados dedicados a la venta de objetos de historia natural, negocios especialmente activos durante la segunda mitad del siglo XIX” (Aragón 105). En Argentina y en Chile, el modelo de la enseñanza secundaria del siglo XIX se fundamentó en la práctica intuitiva basada en el método inductivo, centrado en la observación de la naturaleza, en las colecciones, los museos y laboratorios. En Argentina este modelo se desarrolló de 1870 a 1888, especialmente en los colegios nacionales, que adquirieron gabinetes y libros provenientes de las fábricas y librerías francesas seguidas por las alemanas. Allí se encargaban las colecciones e instrumentos de enseñanza científica. En Chile, la ley de 1883 del gobierno del presidente Domingo Santa María, que preconizaba la reforma pedagógica, permitió invertir presupuesto en la contratación de profesores extranjeros, el fomento de nuevas bibliotecas, así como gabinetes de historia natural. Se destinó "hasta la cantidad de cien mil pesos en la adquisición de mobiliarios, atlas, modelos, y demás utensilios necesarios de enseñanza práctica; i para establecer, de un modo permanente un museo de instrucción primaria” (Anguita citado en Dapelo, Lara y Zumelzu 24). En esa época, Chile importó material didáctico desde Europa y Estados Unidos como una estrategia clave para la enseńanza de las ciencias, debido a la orientación práctica y experimental que los instrumentos, textos y máquinas dieron a la educación de nuestro país desde la segunda mitad del siglo XIX y parte del siglo XX (Dapelo, Lara y Zumelzu 25). En este contexto educativo, Edwin Reed, convencido de la necesidad de contar con gabinetes, señalaba que:

Creo que es tan imposible enseñar historia natural sin una colección de animales y plantas, como enseñar a leer sin libros. Pocos liceos, tal vez ninguno, excepto el de Copiapó tienen colecciones a propósito, y es de lata necesitar tenerlas. 
Propongo hacer unas colecciones [...] Puedo entregar unas cinco colecciones durante el ańo entrante, y cuidaría de entregar los objetos de manera que llegaran al tiempo que se les necesitara en las clases. (Carta de Reed s/f en carta de Philippi al Ministro de Instrucción Pública, 5 diciembre de 1876)

Desde su juventud, Reed manifestó interés en potenciar la enseñanza de las ciencias naturales, lo que hizo como profesor de la disciplina en distintos establecimientos y elaborando diversos manuales, como el "Glosario de términos técnicos de la botánica" (1882), "Compendio de la historia natural de Chile" (1892), y un compendio escolar sobre ciencias físicas y naturales (1893). Estos manuales evidencian el compromiso de Reed con el sistema concéntrico de educación ${ }^{3}$, que buscaba la integración armónica de las materias para que los alumnos avanzaran progresivamente en las asignaturas de acuerdo con el grado de complejidad y el desarrollo de sus capacidades. En 1893, el sistema concéntrico de educación se había aplicado en todas las escuelas públicas del país (Aedo-Richmond 99).

En los años siguientes, Reed seguiría involucrado en la preparación de gabinetes para la venta y también donación. En Concepción, continuó impulsando la formación de gabinetes para la enseñanza de las ciencias naturales, sobre todo valorando las especies nacionales. En 1903 escribe:

Remito a Ud. algunos objetos para el gabinete Escuela superior que Ud. dirige. Mi deseo es formar y suministrar a todas las escuelas del Sur, colecciones escolares en el ramo de Historia Natural que harán posible una enseñanza práctica y rudimentaria de la Zoología y de la Botánica; pero tropiezo con varias dificultades [...] En primer lugar el museo de Concepción se halla escaso de recurso y con un muy reducido personal. Como naturalista y antiguo profesor de las ciencias naturales, se ve que la enseñanza [...] es imposible sin muestras

3 "Como no existe testo, ni programa detallado, para las ciencias físicas i naturales, que se debe enseñar en el primer año, según el nuevo sistema concéntrico, i en la práctica hai muy diversos modos de enseñarlas en los liceos, he escrito este proyecto de testo a fin de uniformar la enseñanza. He tratado de seguir fielmente el proyecto de programa presentado por don Diego Barros Arana al Consejo de Instrucción Pública” (E. Reed Ciencias 4). 
a la vista de los alumnos. Los liceos en general no tienen los gabinetes escolares necesarios para la correcta enseñanza y en cuanto a las escuelas superiores carecen de mucho. Cuando tienen algunos objetos extranjeros y de muy poca utilidad. (Carta de Reed a Juan Miranda, 8 de noviembre de 1903, en C. Reed 41).

Reed también se encontraba interesado en formar gabinetes de historia natural para la enseñanza de las niñas, lo que constituye un aspecto interesante ya que, generalmente, los liceos de niñas se veían en desventaja a la hora de conseguir materiales de este tipo, porque se daba poca importancia a la enseñanza de esta disciplina entre las mujeres. La incorporación de las ciencias en el currículum de los liceos o institutos de enseñanza secundaria femeninos fue ampliamente debatida, porque se consideraba que las mujeres estarían destinadas a cumplir el rol de madres y dueńas de casa, por ello el énfasis estuvo en la educación higiénica y preventiva ${ }^{4}$. Los esfuerzos del naturalista inglés, en este sentido, son destacables para la época. Reed, en carta al Ministro de Instrucción Pública, señala que:

Mi objeto en juntarles ha sido de formar Gabinetes de Historia Natural para los liceos y escuelas fiscales donde estos faltan. Actualmente el Liceo de Niñas de Concepción necesita con urgencia un gabinete y he tomado la libertad de enviar algunas muestras de las más urgentes. Si V.S. lo tiene a bien autorizarme, desde luego puedo mandar al Liceo de Niñas de Concepción más objetos aptos para la enseñanza como también uno que otro objeto al Liceo de Niñas de Talcahuano, donde igualmente un gabinete hace falta. Durante el presente año puedo completar el gabinete para el Liceo de Niñas de Concepción, y tal

$4 \quad$ "Sitúa sus raíces en la influencia pedagógica de la Biología, la Medicina y la Higiene Escolar. Para grandes pedagogos como John Locke (1632-1704), P. Montesino (17811849) o H. Spencer (1820-1903), el primer objetivo de cualquier programa educativo debería consistir en asegurar al educando las cualidades de un buen ser vivo sano. Los enfoques pedagógicos elaborados desde esta perspectiva se preocuparon insistentemente por atender las lacras de una población infantil y adolescente físicamente depauperada y enferma, causada por el tipo de vida impuesto por la sociedad industrial en las grandes urbes, y con el objetivo de regenerar la salud y las fuerzas corporales" (Gómez Gutiérrez 253). 
vez el de Talcahuano [...] sobraran objetos para algunos liceos de la Frontera. (Carta de Reed al Ministerio de Instrucción Pública, 22 de mayo de 1905, en C. Reed 42).

En los años venideros, Edwyn Reed smantuvo su interés en la formación de gabinetes escolares de historia natural. Gracias a la activa interacción que mantuvo con el Liceo de Hombres de Concepción, en 1908 les donó una colección de 287 ejemplares de insectos y compartió públicamente, en carta al rector, la idea de que los museos debían ayudar a la formación de gabinetes para los establecimientos fiscales de instrucción pública en beneficio de la enseñanza de las ciencias naturales en el país.

\section{Reed como director del Museo de Historia Natural DE Concepción}

El Museo de Historia Natural de Concepción fue fundado en 1902 con Edwyn Reed como su primer director, al que se sumó la contratación de Gabriel Castillo como taxidermista. Se trataba del segundo museo de historia natural fundado en una capital regional, tras Valparaíso. Para ese momento, Reed ya era un científico de importante trayectoria: había prestado servicios al Museo Nacional en la clasificación de los insectos y en 1874 tuvo el título de ayudante hasta fines de 1876; había sido director del Museo de Historia Natural de Valparaíso en 1878 y fundador de un gabinete científico en el Seminario San Rafael, entre otras tantas actividades que desarrolló como naturalista.

Los primeros años del museo de Concepción estuvieron ligados al Liceo de Hombres de la ciudad, que le facilitó el espacio para funcionar ${ }^{5}$.

Tanta es la conexión entre el museo y el liceo que, siete años después del fallecimiento de Reed, en 1917, el director del Museo de Historia Natural de Valparaíso, John Juger [lo suponemos por las fechas, aunque no aparece su nombre en el documento], apunta que el Museo de Historia Natural de Concepción ha dejado de existir y funciona como un apéndice del liceo. Esto es desmentido por el rector del Liceo de Hombres de Concepción, don Enrique Molina Garmendia, quien señala que "El Museo de 
Por su parte, Reed arrendó una casa, en calle Chacabuco 29 para preparar los armarios y disponer los ejemplares del museo (C. Reed 15).

Como director, promovió la donación, el canje y la recolección de objetos para la formación de las colecciones que serían parte de la nueva institución. En 1902, encargó ejemplares de toninas a los pescadores de la bahía de Concepción, en Talcahuano, Penco y San Vicente (E. Reed, "Sobre una tunina" 139). Igualmente solicitó diversos peces a los pescadores de Tomé, Lota y Talcahuano, en un esfuerzo por reunir especies regionales. También compró especies locales a los cazadores de la zona (C. Reed, 27).

Reed, junto a la infatigable tarea de organizar las colecciones, se daba tiempo para la investigación científica. A fines de 1902, informaba en la Revista Chilena de Historia Natural, acerca de nuevos insectos chilenos que había observado: "Aunque los múltiples trabajos de la instalación no me han dejado mucho tiempo para la recolección de insectos [...] en el cerro Caracol, de Concepción, he encontrado una especie nueva de mariposa diurna del jénero Neosatyrus en abundancia, una especie nueva de grillo tal vez" (E. Reed, "Nuevos insectos" 20).

Estas labores de observación, las complementaba con el empeño en aumentar la colección. Sobre estos esfuerzos daba cuenta el naturalista y director del Museo de Historia Natural de Valparaíso, Carlos Porter, quien visitó a Reed en el Museo de Concepción, destacando los rápidos progresos que del establecimiento bajo su dirección (Porter 5).

Gracias a la prensa local, que actúa como importante promotora de las actividades vinculadas al museo, podemos conocer la forma en que se exhibían estas primeras colecciones y la disposición de algunos objetos. Esta información fue recopilada en gran parte por el hijo de Edwyn, Carlos Reed, quien también trabajó de forma voluntaria en la institución asistiendo a su progenitor. A un año de la muerte de su padre escribió Apuntes para la

Concepción no es tal 'apéndice' sino que es una institución en cierto sentido público que se halla bajo la supervigilancia del rector del Liceo, está formado casi en su totalidad por objetos que se le han obsequiado por ser de Concepción; se abre al público todos los domingos i cuantas veces lo solicitan de una manera extraordinaria los colejios i los viajeros" (Carta del rector Enrique Molina al ministro de Instrucción Pública, Santiago, 15 de enero 1917). 
historia del Museo de Concepción (1911) con el fin de enaltecer su trayectoria. En esta obra, Carlos Reed recurre tanto a su memoria como a documentos oficiales y publicaciones de los diarios de la época. Gracias a esta publicación podemos conocer información sobre los objetos:

\section{Parte de los objetos colocados en estantes en exhibición en el Liceo (1903)}

\begin{tabular}{lc}
\hline \multicolumn{1}{c}{ Tipo } & Cantidad \\
\hline Mamíferos & 51 \\
Aves extranjeras & 200 \\
Aves chilenas & 400 \\
Reptiles & 20 \\
Peces & 120 \\
Invertebrados & 5.000 \\
Minerales y objetos etnológicos & 300 \\
\hline
\end{tabular}

Fuente: El País, agosto de 1903, en Carlos Reed, Apuntes para la historia del Museo de Concepción (17).

La mayoría de estos objetos fueron adquiridos mediante la caza (mamíferos, aves y peces nacionales), así como por donación y canje con museos del exterior. Las aves extranjeras llegaron mayoritariamente por donaciones, pero también por compras. Respecto de la muestra de aves chilenas, la prensa consideró que era una buena representación de las aves que viven en el centro y sur de Chile y que estaba en buen estado de conservación.

La colección de invertebrados en exposición hacia 1903 correspondía al $82 \%$ de las piezas exhibidas (de un total de 6.091), tal vez porque las especies invertebradas, por sus pequeñas dimensiones, pueden ser mejor exhibidas y dispuestas en los estantes, además del interés que tenía Edwyn Reed, como entomólogo, en ellas ${ }^{6}$.

$6 \quad$ Para el caso del Museo Nacional, Carlos Sanhueza señala que "la materialidad misma de tales objetos permitía su movilidad. La posibilidad de transporte dado su peso ligero (y para el caso de las plantas su embalaje en herbarios), los ponía en ventaja respecto 
En esta publicación de prensa, los minerales y objetos etnológicos aparecen agrupados juntos. Entre los segundos destacan las piedras horadadas chilenas de Quilacoya. En los años siguientes, la colección etnológica tendría un incremento significativo: en 1905 se consigna una momia del Perú, dos cráneos con huesos, una punta de flecha de la isla Mocha y monedas extranjeras. Además de cien fotografías de criminales famosos de la sección antropométrica de la Policía de Santiago (El Sur, 20 de marzo de 1905, en Vergara 41).

Entre la década del veinte y del treinta hubo un incremento de esta sección gracias a la colección de antigüedades diaguitas obtenida por Carlos Oliver Schneider (1899-1949), naturalista, historiador de Concepción y director del Museo de Historia Natural de Concepción (1929-1949). La colección incluía cerámica, artefactos líticos, óseos, metalurgia de cobres, piezas de materialidades mixtas y restos bioantropológicos de 16 individuos (Museo de Historia Natural de Concepción).

Junto a los materiales dispuestos y clasificados en las colecciones, es importante considerar las donaciones, que favorecieron a un museo de escaso presupuesto en sus orígenes. La identificación de los donadores puede constituir el punto de partida para seguir la historia de los objetos y comprender las relaciones que se establecen entre el donador y el museo. En este aspecto, la prensa local de la época da cuenta al menos de algunas donaciones recibidas por la institución ${ }^{7}$ :

de otras especies. En otro sentido, las posibilidades de conservación en condiciones de movilidad precarias, les hacía ideales para su recolección” (“Objetos” 152).

De acuerdo con Leoncio López-Ocón, los filántropos que donan a la ciencia "expresan un interés por lo propio, un sentimiento de pertenencia local. Gran parte de esas donaciones desean contribuir a la perfección del inventario de los recursos propios del territorio y a hacer posible un uso racional de ellos [...] crean entonces un patrimonio que se hereda, se gestiona y se transmite, que habla de pasado, se vive en el presente y en el futuro" (413). 
Principales donadores diciembre 1907

\begin{tabular}{ll}
\hline \multicolumn{1}{c}{ Donador } & \multicolumn{1}{c}{ Objeto } \\
\hline Fernando Paulsen & Picaflor de Juan Fernández \\
Enrique Merino Reyes & Bala de cobre (antigua) encontrada en Talcahuano \\
Ángel Penco & Un ave exótica \\
Sr. Sayago & Una esponja marítima de San Vicente \\
Federico Videla & Cuatro aves: dos cuervos y dos pajaritos "escasos en \\
& el sur" \\
Isidro Villarroel & Dos llamas vivas \\
\hline
\end{tabular}

Fuente: El Sur, 30 de diciembre de 1907 en Carlos Reed, Apuntes para la Historia del Museo de Concepción (25).

El picaflor donado por Fernando Paulsen resulta de interés para el museo ya que se trata de una especie que, en la época, ya estaba considerada en peligro de extinción ${ }^{8}$. Paulsen también donó a la sección zoológica del Museo de Historia Natural de Valparaíso, al igual que Federico Videla. Con respecto a las llamas donadas por Isidro Villarroel, el director dejó una para embalsamar y otra para ser exhibida en el jardín zoológico de la quinta agrícola.

Reed, al igual que Carlos Porter en Valparaíso, tiene una política activa de canjes de objetos con museos extranjeros: objetos de esquimales y pieles rojas enviados por el Museo Nacional de Estados Unidos (C. Reed 66), aunque la disgregación física del propio museo dificulta la conservación

8 "Según él [Fernando Paulsen] solamente existe en aquella isla y con la colonización es probable que en pocos años más esté estinguida. Según opinión de los naturalistas de más fama, el Picaflor de la isla y la especie común de Chile han tenido un antepasado común. La especie de Juan Fernández encontró clima más a propósito y alimentación más abundante [...] lo que más llama la atención es la marcada diferencia en el plumaje de los dos sexos, tan diverso que casi todos los naturalistas extranjeros los han considerado como dos especies distintas. En la obra de Gay están descritas [...] la isla, después, los naturalistas han dicho que deben ser los sexos de una sola especie. En 1872 el Señor Reed visitó la isla, mató unos 5 ejemplares e hizo un cuidadoso 'post mortum' en cada uno, de sus estudios resultó que las supuestas dos especies eran una sola y que todos los ejemplares bermejos, con nuca cobriza, eran machos y los verdosos eran hembras" (C. Reed 25). 
y seguimiento de las piezas9 (algo similar a lo que enfrentó el Museo de Historia Natural de Valparaíso en sus inicios).

A pesar de las dificultades que enfrenta el museo de Concepción, como la falta de espacio y de local y las carencias económicas, Edwyn Reed valora sus colecciones. Señala que son extensas y valiosas, comparables a las del museo de Valparaíso antes del terremoto (ocurrido en 1906) o a las del museo de Santiago antes de su traslado a la Quinta Normal. Reed cuenta con un presupuesto limitado para comprar piezas; en 1908 seńala que ya no puede comprar en Europa por la baja del cambio y la reducción del presupuesto. En 1910 hace un inventario de las piezas; destaca entre ellas "1 hiena, 1 lobo europeo, 1 zorro amebiano, 2 topos europeos, 1 musaraña, 2 erizos de Europa, 1 tigre africano, 1 zorro europeo, 1 tejón, 14 mamíferos exóticos, 1 momia peruana, 48 frascos con preparaciones y 50 objetos osteológicos diversos" (Inventario de 1910, en Vergara 43). Lo diverso del inventario da cuenta del esfuerzo de Reed por conseguir especies y objetos con el fin pedagógico de acercar a la población al conocimiento de la historia natural, manifestando en el diario El Sur que entre el público que visita el museo hay un

crecido número de artesanos, de nińos de las escuelas primarias y personas de pueblo que, satisfaciendo una curiosidad muy explicable, adquieren objetivamente algunas importantes nociones que de otro modo tal vez nunca llegarían a poseer. La sala que ayer se inauguró tuvo 283 visitantes y todo el museo más de 500. (El Sur, 30 de diciembre de 1907, en C. Reed 21)

De esta manera, las colecciones gestionadas por Edwyn Reed fomentan el conocimiento de las diversas especies zoológicas nacionales y regionales, así como también de la flora chilena. También hay antigüedades

9 El 30 de diciembre de 1907, el diario El Sur señala que "El Museo de Concepción se encuentra diseminado en tres puntos distintos: en una sala del liceo de hombres, en varias salas de la casa particular del director y en una bodega ruinosa que se ha logrado habilitar recientemente". En 1908, Reed expone al ministro la necesidad de un cambio de local: la colección ha crecido mucho y el liceo se ha convertido en un lugar inadecuado (C. Reed 25). 
prehispánicas que comparten espacio con las especies naturales provenientes desde distintos puntos del globo. Esto refleja no solo un acopio de materiales, sino de saberes dispuestos para su interpretación, con el fin de fomentar la investigación, la reflexión y la difusión del conocimiento científico a nivel regional y nacional, evidenciando también las importantes redes de colaboración entre naturalistas y la comunidad, que permiten finalmente consolidar el Museo de Historia Natural de Concepción.

\section{Conclusiones}

Las investigaciones en torno a la figura de los naturalistas y sus redes colaborativas permiten reconstruir en parte las historias sobre la formación de las colecciones de los museos de historia natural en Chile, una labor ya iniciada por investigadores nacionales (Sanhueza, Vilo, Serra, Saldivia y Urizar, entre otros), y que tiene una tradición consolidada en América Latina (Podgorny y Lopes, Mayoni, Gänger, Nieto Olarte). No obstante, es fundamental continuar con estas investigaciones históricas, pues aún no hay suficientes estudios que pongan en valor las colecciones de los museos de Valparaíso y de Concepción, así como la labor específica de cada uno de sus actores ${ }^{10}$.

Las labores desempeñadas por Edwyn Reed en Chile durante su larga trayectoria como naturalista es continuidad de los trabajos emprendidos por Claudio Gay y Rodulfo Philippi en esta materia, a pesar de los problemas internos que alejarían a Reed del director del Museo Nacional en Santiago. Es importante destacar que Edwyn Reed exploró el territorio nacional y clasificó diversas especies de la flora y la fauna en un claro esfuerzo de continuidad de la labor de clasificación hecha por Claudio Gay. Asimismo,

10 En esta materia, ha habido avances importantes. Durante 2019 se presentó una exposición en la Galería Patrimonio Chileno del Centro Cultural La Moneda que recorre los 140 ańos del Museo de Historia Natural de Valparaíso, el primer museo regional del país. La exposición se titula "Maravillas, colecciones e historias" (Ministerio de las Culturas, Las Artes y el Patrimonio). 
actuó como agente activo en el canje de objetos y conocimiento con Europa y, gracias a su experiencia, estableció diversos museos regionales al organizar las colecciones de historia natural, tarea realizada años antes por Rodulfo Philippi y, posteriormente, por su hijo Federico en la capital.

Edwin Reed formó parte activa de las redes de intercambio científico a escala global, relacionándose con naturalistas chilenos y extranjeros. Por ello, el acopio de objetos para el Museo de Historia Natural de Concepción fue facilitado por las donaciones de particulares e instituciones, así como por el canje con museos del mundo. La compra también fue un medio de adquirir objetos, a pesar del limitado presupuesto anual, que debía cubrir la necesidad de materiales para la preservación de las colecciones, así como los sueldos de los funcionarios. La donación y el canje fueron medios importantes para formar las colecciones de historia natural, no tan solo en Concepción, sino en Chile durante fines del siglo XIX y principios del XX.

A lo largo de su vida profesional, Edwyn Reed se comprometió con la enseñanza de las ciencias naturales, lo que se expresó en la elaboración de manuales escolares y en la creación de gabinetes para la enseñanza. Este interés se enmarca en la convicción de que el aprendizaje de las ciencias naturales conlleva la adquisición de valores para la juventud como la sencillez, la disciplina y el autocontrol. A principios del siglo XX, Reed también confeccionó gabinetes para los liceos de niñas en una época donde la disciplina no era considerada de gran valor en su formación. La elaboración de estos gabinetes evidencia un compromiso de los naturalistas con la enseñanza práctica de las ciencias naturales en los principales establecimientos secundarios del país, lo que a su vez consolida a estos museos como centros de difusión del conocimiento científico.

La labor de Reed en la formación del Museo de Historia Natural de Concepción se complementa con su labor como científico en la identificación de especies chilenas. A estas actividades estuvo dedicado desde el tiempo de sus exploraciones en Brasil y luego en Chile, donde estudió y publicó sobre diversas especies. Este gran conocimiento le proporcionó la experiencia que, décadas más tarde, se reflejó en el éxito de la organización del museo en Concepción. 
Desafortunadamente, es difícil seguir el rastro de las redes de colaboración que tuvo Reed en la clasificación de especies y en la formación de los museos y gabinetes escolares. Sabemos que contó con una red local que lo apoyó en la recolección de objetos, entre ellos, pescadores, cazadores o naturalistas aficionados. Sin embargo, en los documentos oficiales sus nombres están silenciados y, por tanto, la visibilización de estos agentes es escasa, constituyendo un problema histórico para futuras investigaciones.

La contribución de Edwyn Reed va más allá de la formación de las colecciones de historia natural a nivel regional. Las evidencias presentadas nos permiten observar su contribución al desarrollo de las ciencias naturales en Chile, pero también un trabajo activo y colaborativo donde la ciudadanía tiene un papel importante como donantes de objetos, muchos de los cuales constituyen el origen de las actuales colecciones del Museo de Historia Natural de Concepción.

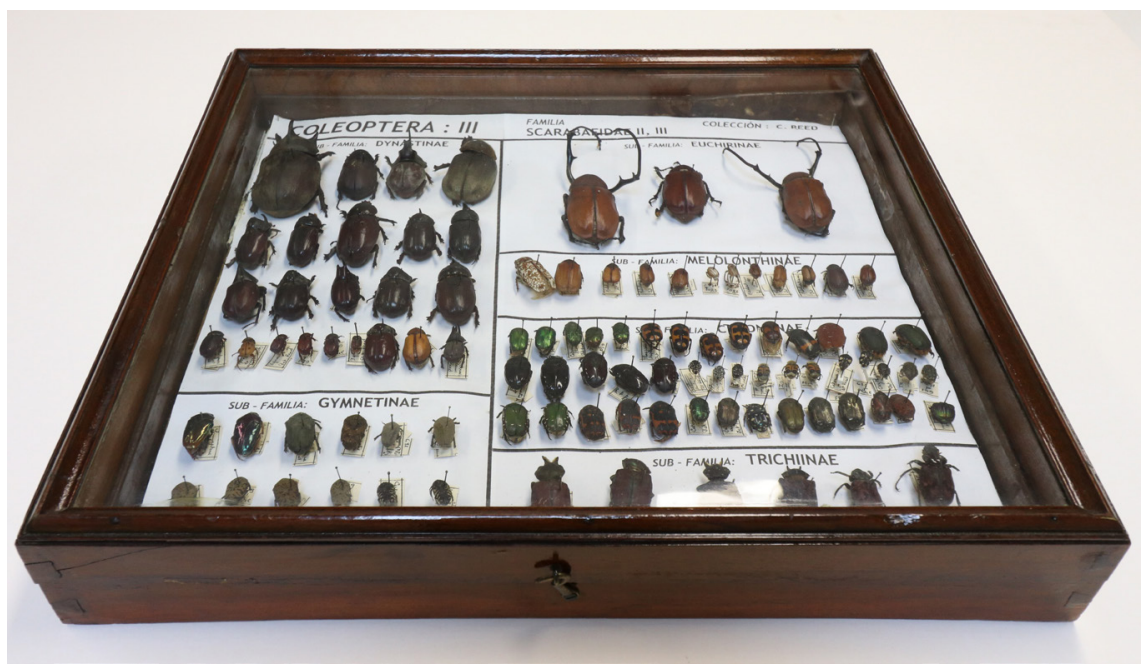




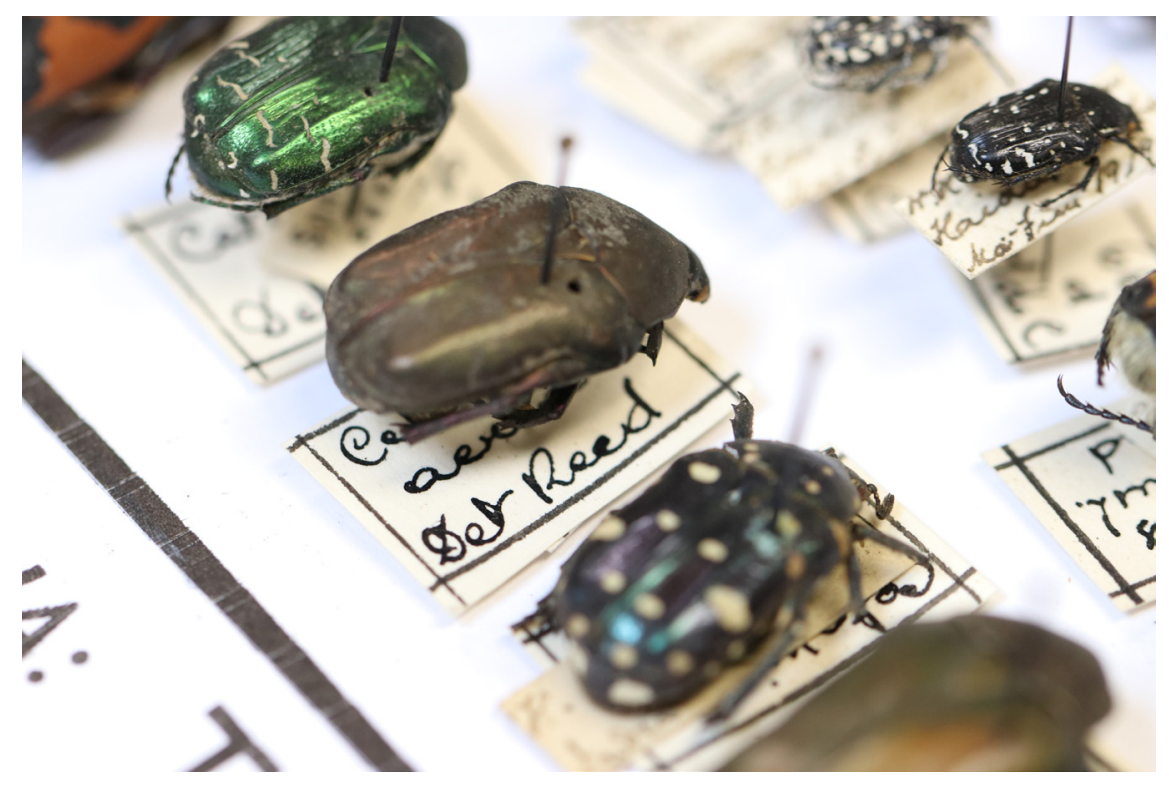

Fuente: Colección entomológica del naturalista Carlos Reed, hijo de Edwyn Reed, Museo de Historia Natural de Concepción. 


\section{Bibliografía}

Aedo-Richmond, Ruth. La educación privada en Chile: un estudio histórico-analitico desde el periodo colonial hasta 1990. Santiago de Chile: Ril, 2000.

Alberti, Samuel. Nature and Culture. Objects, Disciplines and the Manchester Museum. Manchester: Manchester University Press, 2009.

Aragón Albillos, Santiago. "Historias de objetos que cuentan historias: plantas, rocas y animales en los institutos históricos madrileños". Aulas con memoria. Ciencia, educación y patrimonio en los institutos históricos de Madrid (18371936). Editado por Leoncio López-Ocón, Santiago Aragón y Mario Pedrazuela. Madrid: CEIMES / CSIC, 2012, pp. 105-16.

Carta de R. A. Philippi al Ministerio de Instrucción Pública, Santiago 3 de agosto de 1870. Archivo Ministerio de Educación, vol. 138, documento 35, foja 3.

Carta de R. A. Philippi al Ministro de Instrucción Pública. Santiago, 13 de mayo de 1872. Archivo Ministerio de Educación, vol. 138, documento 42, foja 1.

Carta de Edwyn Reed s/f en Carta de R. A. Philippi al Ministro de Instrucción Pública, Santiago, 5 diciembre de 1876. Archivo Ministerio de Educación, vol. 138. documento 77, foja 1 .

Carta del Rector Enrique Molina al Sr. Ministro de Instrucción Pública. Concepción, 15 de enero de 1917. Archivo Liceo Enrique Molina Garmendia, impreso foja 4.

Dapelo, Gonzalo, Mario Lara y Thamara Zumelzu. Enseñanza científica e identidad nacional, revalorización de la enseñanza práctica experimental: el caso del Liceo de Hombres de Talca. Tesis de Licenciatura, Universidad Autónoma de Chile, 2019.

Gänger, Stefanie. Relics of the past. The Collecting and Study of Pre-Columbian Antiquities in Peru and Chile, 1837-1911. Oxford: Oxford University Press, 2014.

García, Susana y María Gabriela Mayoni. "Los museos y gabinetes de ciencias en los colegios nacionales de la Argentina (1870-1880)”. Boletín del Instituto de Historia Argentina y Americana, n. ${ }^{\circ}$ 50, 2019, pp. 135-62.

Garrido, Francisco. "Rodulfo Philippi y la protección del patrimonio cultural en Chile", Museo Nacional de Historia Natural, 4 enero 2019, www.mnhn.gob. cl. 
Gómez Gutiérrez, Juan Luis. “Naturaleza versus educación: análisis de las experiencias educativas que tuvieron en la naturaleza su principal escenario (siglos XIXXX)”. Social and Education History, vol. 8, n. ${ }^{\circ}$ 3, 2019, pp. 249-71.

López-Ocón, Leoncio. "Los museos de historia natural en el siglo XIX: templos, laboratorios y teatros de la naturaleza". Arbor, vol. 163, n. ${ }^{\circ}$ 643-644, 1999, pp. 409-23.

Ministerio de las Culturas, Las Artes y el Patrimonio. "Muestra recorre los 140 años del primer museo regional del país”, www.patrimoniocultural.gob.cl.

Museo de Historia Natural de Concepción. "Colección Diaguita: su viaje desde el Norte Chico hasta Concepción”, 21 de octubre de 2018, www. museodehistorianaturaldeconcepcion.cl.

Oficios enviados al Museo Nacional. Santiago: 1843-1900. Ministerio de Instrucción Pública. Archivo Ministerio de Educación.

Philippi, Federico. Historia del Museo Nacional de Chile por el Dr. R. A. Philippi. Boletín del Museo Nacional. Tomo I. Santiago de Chile: Imprenta, Litografía y Encuadernación Barcelona, 1908.

Plan de Estudios y programas de Instrucción Secundaria aprobados por el Consejo de Instrucción Pública para los liceos del Estado, Santiago de Chile: Imprenta Cervantes, 1893. Biblioteca Nacional, www.bibliotecanacionaldigital.gob.cl

Planes y métodos de estudio. Memoria Chilena, www.memoriachilena.gob.cl

Podgorny, Irina. "Bureaucracy, Instructions and Paperwork- The Gathering of Data about the Three Kingdoms of Nature in the Americas, 1770-1815”. Nuevo Mundo, Mundos Nuevos, 2019, https://journals.openedition.org/ nuevomundo/75454.

Podgorny, Irina y María Margarita Lopes. El desierto en una vitrina: museos e historia natural. México: Limusa, 2008.

Porter, Carlos. "Galería de Naturalistas de Chile: Don Edwyn C. Reed". Revista Chilena de Historia Natural, año VII, n.o 3, 1903, pp. 138-40.

Reed, Carlos. Apuntes para la historia del Museo de Concepción. Buenos Aires: Establecimiento Gráfico Olivetti Hermanos, 1911.

Reed, Edwyn C. Ciencias físicas y naturales. 1er Año. Adaptado al programa del primer año de estudios de instrucción secundaria, presentado al Consejo de Instrucción Pública por don Diego Barros Arana. Santiago de Chile: Imprenta Gutenberg, 1893. 
_. "Nuevos insectos chilenos". Revista Chilena de Historia Natural, año VI, 1902, p. 20.

_. "Sobre una tunina chilena". Revista Chilena de Historia Natural, año VIII, 1904, pp. 138-39.

Reed, Walter. Edwyn C. Reed y el archivador rojo. Madrid: Publicación de Rodrigo Quezada Reed, 2013.

Revista Chilena de Historia Natural, año VI-IX (1902-1905).

Sanhueza, Carlos. "Coleccionismo en el Museo Nacional de Chile (1853-1897)". La movilidad del saber científico en América Latina. Objetos, prácticas e instituciones (siglos XVIII al XX). Editado por Carlos Sanhueza. Santiago: Editorial Universitaria, 2018, pp. 169-96.

_. $\quad$ "Objetos naturales en movimiento. Acerca de la formación de las Colecciones del Museo Nacional de Chile (1853-1897)". Revista de Humanidades, n. ${ }^{\circ} 34$, 2016, pp. 143-69.

Schell, Patience A. "Natural History Values and Meanings in Nineteenth-Century Chile". Notes and Records. Reino Unido: The Royal Society Publishing, 2018, pp. 1-25.

Serra, Daniela. "La configuración de un espacio para el saber natural en Chile. El Gabinete de Historia Natural de Santiago, 1830-1842". Tendencias y perspectivas de la cultura cientifica en Chile y América Latina. Siglos XIXXXI. Editado por Carolina Valenzuela. Santiago: Ril, 2019, pp. 51-76.

_. $\quad$ "Minerales y colección en el reino de Chile. El Gabinete de la Real Academia de San Luis, 1790-1810". Historia Unisinos, vol. 23, n. ${ }^{\circ} 2$, 2019, pp. 15666.

Vergara Aravena, José. "El Museo de Concepción: reseña de noventa años". Comunicaciones del Museo de Concepción, n. ${ }^{\circ}$ 7, 1993, pp. 63-90.

Vilo Muñoz, Felipe y Carlos Sanhueza Cerda. "Comunidades en movimiento. La circulación de las obras zoológicas de Rudolph Philippi en Chile (18531904)". Historia 396, vol. 7, n. ${ }^{\circ}$ 2, 2017, pp. 597-25. 